\title{
Recombinant GII.4[P31] Was Predominant Norovirus Circulating in Beijing Area, China, 2018-2020
}

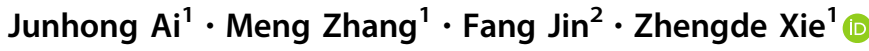

Received: 28 November 2020 / Accepted: 20 February 2021 / Published online: 9 April 2021

(c) Wuhan Institute of Virology, CAS 2021

\section{Dear Editor,}

Acute gastroenteritis (AGE) is a leading infectious cause of morbidity worldwide, particularly among children in developing countries (Mortality and Causes of Death 2016). With the introduction of rotavirus vaccines, noroviruses have been identified as a leading cause of AGE outbreaks and sporadic disease worldwide (Hall et al. 2013). The norovirus genome contains three open reading frames (ORFs). ORF1 encodes the nonstructural viral proteins including the RNA-dependent RNA polymerase. ORF2 and ORF3 encode the respective major (VP1) and minor (VP2) structural proteins. Based on phylogenetic clustering of the complete VP1 amino acid sequence, norovirus can be divided into 10 genogroups $(\mathrm{G})$ (Chhabra et al. 2019) of which GI, GII, and GIV have been found among human beings. Genogroups can be subdivided into 9 GI, 27 GII, and 2 GIV genotypes (Chhabra et al. 2019). A dual-nomenclature system has been proposed for GI and GII noroviruses using partial regions of the ORF1 RNA polymerase $(\mathrm{P})$-encoding region and partial region of ORF2.

Since the mid-1990s, genogroup II genotype 4 (GII.4) noroviruses have caused the majority of outbreaks and sporadic cases worldwide. Recombination which often occurs at the ORF1/ORF2 junction and antigenic drift are

Zhengde Xie

xiezhengde@bch.com.cn

1 Beijing Key Laboratory of Pediatric Respiratory Infection Diseases, Key Laboratory of Major Diseases in Children, Ministry of Education, National Clinical Research Center for Respiratory Diseases, Research Unit of Critical Infection in Children, Chinese Academy of Medical Sciences, 2019RU016, Laboratory of Infection and Virology, Beijing Pediatric Research Institute, Beijing Children's Hospital, Capital Medical University, National Center for Children's Health, Beijing 100045, China

2 Department of Laboratory Medicine, Beijing Children's Hospital, Capital Medical University, National Center for Children's Health, Beijing 100045, China the mechanism of evolution for norovirus. New GII.4 variants emerged with 2-3-year periodicity up to 2012. Afterwards, the GII.4 Sydney capsid seems to persist through recombination, with a novel recombinant of GII.4 Sydney[P16] detected in 2014 in Germany and the Netherlands, and again in 2016 in Japan, China, and the Netherlands (van Beek et al. 2018). However, there were few studies about the genotype of norovirus in sporadic gastroenteritis cases in China, recently. Here, we describe the identified genotype of norovirus in sporadic AGE cases in Beijing area, China.

From November 2018 to September 2020, a prospective study about viral etiology of AGE in pediatric outpatients was conducted in Beijing Children's Hospital. AGE was defined as $>3$ events involving loose feces, vomiting, or both within a $24-\mathrm{h}$ period. A total of 605 patients each one with a fecal sample were enrolled into this study. Among them, $64 \%$ were male, $36 \%$ were female. The sex ratio (male/female) was 1.78:1. The age of patients ranged from one month to 16.0 years (median, 1.0 year). About $64.1 \%$ (388/605) cases were infants less than 1 year old.

Viral RNA was extracted from $10 \%$ fecal suspension (mixing $0.1 \mathrm{~g}$ feces with $1.0 \mathrm{~mL}$ phosphate-buffered saline ( $\mathrm{pH}$ 7.2)). Then a commercial kit of multiplex real-time PCR for norovirus (GI and GII) and rotavirus (group A) (Shuoshi, Jiangsu, China) was used in this study. The positive samples of norovirus were amplified by conventional RT-PCR with SuperScript ${ }^{\mathrm{TM}}$ III One-Step RT-PCR System with Platinum ${ }^{\mathrm{TM}}$ Taq DNA Polymerase kit (Invitrogen). Dual-typing system of norovirus was used in this study, which amplifies a partial region of ORF1 and a partial region of ORF2 (van Beek et al. 2018). The previously published oligonucleotide primers for GII viruses were MON431 (TGGACIAGRGGICCYAAYCA) and G2SKR (CCRCCNGCATRHCCRTTRTACAT). The expected PCR product size is 570 bp (Cannon et al. 2017, 2019). All PCR products were subjected to Sanger sequencing and genotypes were assigned using the Norovirus Genotyping tool (http:// www.rivm.nl/mpf/norovirus/typingtool). A phylogenetic tree 
was constructed using the maximum likelihood method implemented in MEGA software (version 5.01) with 1000 bootstrap replicates. The sequences of the noroviruses identified in this study were deposited in GenBank (GenBank accession numbers: MW205541-MW205668).

Overall, norovirus was identified in $30.7 \%$ (186/605) of all tested samples. The AGE patients were divided into three age groups: $0-1$ year $(n=388), 2-5$ years $(n=126)$ and $6-18$ years $(n=91)$. The positive rate of norovirus was $34.3 \%$ in $0-1$ year age group, followed by $27.0 \%$ and $20.9 \%$ in $2-5$ years age group and $6-18$ years age group, respectively. There were significant differences in the positive rate of norovirus between different age groups $(P=0.026)$. Young children are the risk groups of norovirus infection. As in this study, the detection rate of norovirus in young children is relatively high, especially in children under 1 year old.
The epidemic of norovirus is seasonal. Most outbreaks occurred during the winter season (November-March) (Jin et al. 2020). In this study, norovirus positive cases were also mainly in winter, accounted for $76.34 \%(142 / 186)$ of all positive cases, which is consistent with the seasonal distribution of norovirus.

For norovirus positive cases, most of them were GII strain with a positive rate of $95.7 \%(178 / 186)$. GI strain was only detected in $9.1 \%(17 / 186)$ cases. There were also $4.8 \%(9 / 186)$ cases with a co-infection of GI and GII strains.

Among the GII positive cases, 128 were further genotyped. GII.P31 $(52.3 \%, 67 / 128)$ was the most common RdRp genotype, followed by GII.P16 $(28.1 \%, 36 / 128)$ and GII.P12 (14.8\%, 19/128) (Fig. 1A). For VP1 genotype, the three most common were GII.4 $(68.8 \%, 88 / 128)$, GII.3 $(14.8 \%, 19 / 128)$ and GII.2 (11.7\%, 15/128) (Fig. 1B). The most prevalent strain was recombinant GII.4[P31] (52.3\%,
A

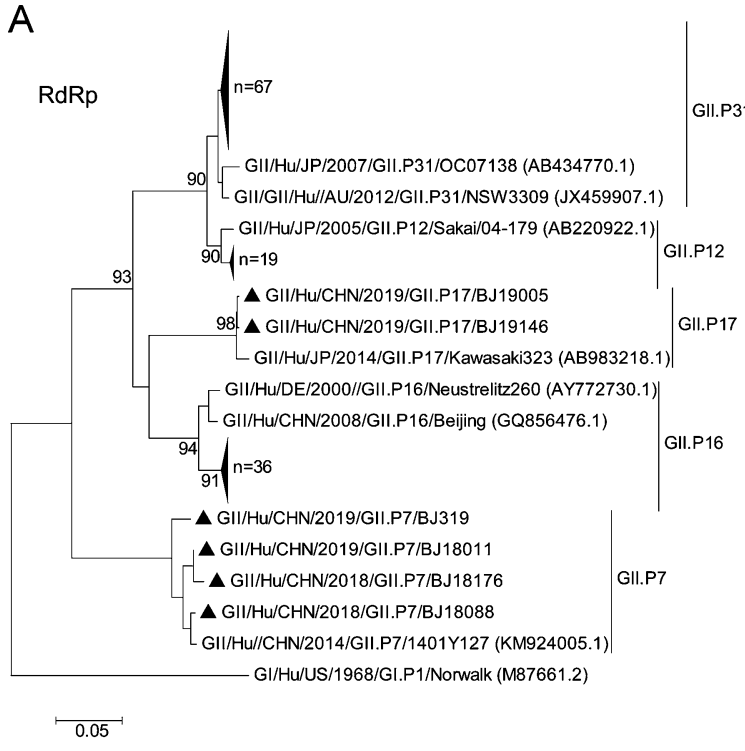

B

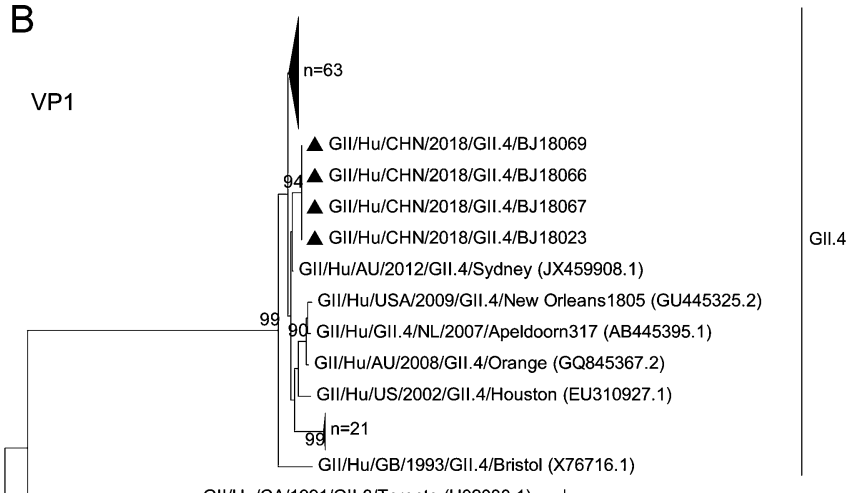

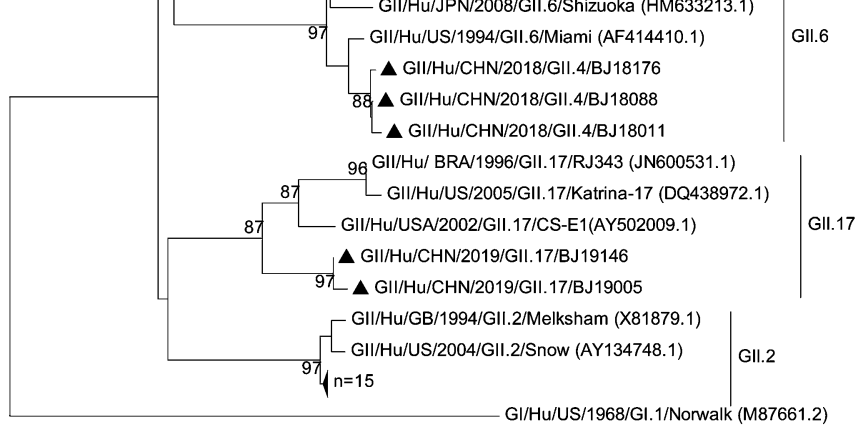

$\stackrel{\vdash}{\square .1}$

Fig. 1 Phylogenetic trees of the norovirus GII partial-nucleotide sequences. A Analysis of the RNA-dependent RNA polymerase $(R d R p)$ region $(251 \mathrm{bp})$. B Analysis of the major capsid protein VP1 region (302 bp). Trees were generated by using the Neighbor-Joining method with 1,000 bootstrap replicates implemented in MEGA5 (https://www.megasoftware.net). Bootstrap values $>80$ are indicated at the nodes. Strains of sufficient nucleotide sequence length were included in the trees (denoted individually with black triangles and in groups with large triangles). Reference strains are shown with accession numbers (in parentheses). Scale bars indicate nucleotide substitutions per site 
67/128), followed by GII.4[P16] (16.4\%, 21/128), GII.3[P12] (14.1\%, 18/128) and GII.2[P16] (12.5\%, 16/128). GII.17[P17] norovirus was only detected in $1.6 \%$ $(2 / 128)$ cases. However, the rare recombinant norovirus genotype GII.6[P7] in China was found in 3.1\% (4/128) cases here.

Norovirus continues to evolve through genetic mutation and recombination. It is reported that, the prevalent genotype of norovirus circulating in the same region will change every several years (Hasing et al. 2019). Even in the same period, the genotypes of norovirus circulating in different regions may be different. In this study, the most prevalent norovirus was recombinant GII.4[P31] genotype in sporadic AGE children. It is similar to the study of sporadic cases in Shanghai from 2014 to 2018, in which the predominant genotype was GII.4 Sydney[P31] (Wang et al. 2019). However, it is different with norovirus outbreaks studies in the past 5 years. In China, the predominant genotype of norovirus outbreaks were GII.17[P17] and GII.2[P16] in 2014-2015 and 2016-2018, respectively (Ao et al. 2017; Gao et al. 2019; Jin et al. 2020).

In this study, the most prevalent genotype was recombinant GII.4[P31]. It is reported that over the past 10 years, GII.4 Sydney[P31] and GII.4 Sydney[P16] were the predominant strain epidemic globally (van Beek et al. 2018). GII.4 Sydney[P31] has been the predominant strain in Canada (Hasing et al. 2019) and Thailand (Chuchaona et al. 2019) since 2012. And GII.4 Sydney[P16] has been popular in Germany, Netherlands, America, Japan and Canada since 2014 (Cannon et al. 2017; van Beek et al. 2018; Hasing et al. 2019).

In conclusion, norovirus was identified in $30.7 \%$ of sporadic AGE children. And the most prevalent genotype was recombinant GII.4[P31] in Beijing area in 2018-2020. Norovirus is evolving by recombination and antigenic drift. Thus, continuous monitoring of the circulating genotypes in pediatric population is needed for current norovirus vaccine development.

Acknowledgements This study was approved by the Medical Ethics Committee of the Beijing Children's Hospital, Capital Medical University, and informed consent was obtained. We would like to acknowledge all the physicians and participants for collecting clinical specimens from the patients used in this study. We thank all the reviewers for comments that improved this manuscript. This work was supported by grants from National Major Science \& Technology Project for Control and Prevention of Major Infectious Diseases in China (2018ZX10201002-009-005).

\section{Compliance with Ethical Standards}

Conflict of interest The authors declare that they have no conflict of interests.
Human and Animal Rights Statement This study conformed to the 1975 Declaration of Helsinki guidelines and was approved by Medical Ethics Committee of Beijing Children's Hospital, Capital Medical University (2018-k-147).

\section{References}

Ao Y, Wang J, Ling H, He Y, Dong X, Wang X, Peng J, Zhang H, Jin M, Duan Z (2017) Norovirus GII.P16/GII.2-associated gastroenteritis, China, 2016. Emerg Infect Dis 23:1172-1175

Cannon JL, Barclay L, Collins NR, Wikswo ME, Castro CJ, Magana LC, Gregoricus N, Marine RL, Chhabra P, Vinje J (2017) Genetic and Epidemiologic Trends of norovirus outbreaks in the United States from 2013 to 2016 demonstrated emergence of Novel GII.4 recombinant viruses. J Clin Microbiol 55:2208-2221

Cannon JL, Barclay L, Collins NR, Wikswo ME, Castro CJ, Magana LC, Gregoricus N, Marine RL, Chhabra P, Vinje J (2019) Correction for Cannon et al., genetic and epidemiologic trends of norovirus outbreaks in the United States from 2013 to 2016 demonstrated emergence of novel GII.4 recombinant viruses. J Clin Microbiol 57:e00695-19

Chhabra P, de Graaf M, Parra GI, Chan MC, Green K, Martella V, Wang Q, White PA, Katayama K, Vennema H, Koopmans MPG, Vinje J (2019) Updated classification of norovirus genogroups and genotypes. J Gen Virol 100:1393-1406

Chuchaona W, Chansaenroj J, Wanlapakorn N, Vongpunsawad S, Poovorawan Y (2019) Recombinant GII.Pe-GII.4 Norovirus, Thailand, 2017-2018. Emerg Infect Dis 25:1612-1614

Gao Z, Liu B, Yan H, Li W, Jia L, Tian Y, Chen Y, Wang Q, Pang X (2019) Norovirus outbreaks in Beijing, China, from 2014 to 2017. J Infect 79:159-166

Hall AJ, Lopman BA, Payne DC, Patel MM, Gastanaduy PA, Vinje J, Parashar UD (2013) Norovirus disease in the United States. Emerg Infect Dis 19:1198-1205

Hasing ME, Lee BE, Qiu Y, Xia M, Pabbaraju K, Wong A, Tipples G, Jiang X, Pang XL (2019) Changes in norovirus genotype diversity in gastroenteritis outbreaks in Alberta, Canada: 2012-2018. BMC Infect Dis 19:177

Jin M, Wu S, Kong X, Xie H, Fu J, He Y, Feng W, Liu N, Li J, Rainey JJ, Hall AJ, Vinje J, Duan Z (2020) Norovirus Outbreak Surveillance, China, 2016-2018. Emerg Infect Dis 26:437-445

GBD Mortality Causes of Death C (2016) Global, regional, and national life expectancy, all-cause mortality, and cause-specific mortality for 249 causes of death, 1980-2015: a systematic analysis for the Global Burden of Disease Study 2015. Lancet 388:1459-1544

van Beek J, de Graaf M, Al-Hello H, Allen DJ, Ambert-Balay K, Botteldoorn N, Brytting M, Buesa J, Cabrerizo M, Chan M, Cloak F, Di Bartolo I, Guix S, Hewitt J, Iritani N, Jin M, Johne R, Lederer I, Mans J, Martella V, Maunula L, McAllister G, Niendorf S, Niesters HG, Podkolzin AT, Poljsak-Prijatelj M, Rasmussen LD, Reuter G, Tuite G, Kroneman A, Vennema H, Koopmans MPG, NoroNet, (2018) Molecular surveillance of norovirus, 2005-16: an epidemiological analysis of data collected from the NoroNet network. Lancet Infect Dis 18:545-553

Wang X, Wei Z, Guo J, Cai J, Chang H, Ge Y, Zeng M (2019) Norovirus activity and genotypes in sporadic acute diarrhea in Children in Shanghai during 2014-2018. Pediatr Infect Dis J 38:1085-1089 